\title{
The effect of material uncertainties on envelope heat transfer simulations
}

\author{
Alessandro Prada - Free University of Bolzano, Bolzano, Italy \\ Paolo Baggio - Free University of Bolzano, Bolzano, Italy \\ Marco Baratieri - Free University of Bolzano, Bolzano, Italy \\ Andrea Gasparella - Free University of Bolzano, Bolzano, Italy
}

\begin{abstract}
In this paper, the influence of thermo-physical properties on the heat flux transmitted through the envelope is investigated. In fact, the retrieval of reliable thermophysical properties is one of the major difficulties in the early stage of energy simulations. This issue is further emphasized if the model refers to existing buildings, when usually reference thermo-physical properties are used in lieu of declared and certified values (i.e. $90 \%$ fractile). Moreover, the thermal characteristics, especially for porous media, are closely related to the water content and, to a lesser extent, to temperature and age. In addition to this, the uncertainties due to simplifying assumptions, commonly made in thermal analysis, should not be forgotten. For instance, the material apparent conductivities, as well as the other thermophysical properties, are the macroscopic results of various basic mechanisms such as the solid and gas conduction, the gas convection and the long wave radiation.

The question then addresses to what extent the uncertainties of thermo-physical properties affect the reliability of the heat transfer through envelope and, consequently, of the energy simulation predictions. In order to answer this question, an uncertainty analysis has been carried out by means of a Monte Carlo approach. This procedure is applied for the recursive numerical solution of the partial differential equation of the heat conduction.

Different Italian climates (i.e. Trento and Palermo) and typical wall typologies are adopted in order to broaden the representativeness of the results. Furthermore, two methods widely used in hourly simulation code (i.e. Conduction Transfer Function and Finite Difference Method) are herein analysed.
\end{abstract}

\section{Introduction}

Dynamic energy simulation has the potential to provide relevant information about the building energy behaviour and to indicate the possible conservation measures for the reduction of energy consumption. In fact, these enhanced methods allow for better modelling of the dynamic interactions between building, occupants and energy systems. On the other hand, one of the problems in the application of enhanced simulation models, which can sometimes undermine the reliability of their results, is the difficulty to gather reliable input data. The reliability of simulation outcomes hinges upon the accuracy of input data, simulation models and energy modellers all together. Without these characteristics, significant deviations between the actual and the simulated energy performance can be found. Therefore, an estimation of the sensitivity and of the degree of uncertainty introduced by each factor can help to increase the awareness of the result reliability and of the robustness of the whole simulation process. In the last few years, increasing attention has been paid to the uncertainty and sensitivity analyses on building energy simulations. In one of the earliest works (Lomas and Eppel, 1992), the authors compared three different techniques for sensitivity analysis. Following on from this work, Macdonald (Macdonald, 2002) integrated some uncertainty procedures in the software Esp-r. In addition, in (Costola, Blocken and Hensen, 2009) the effect of uncertainties in wind-pressure coefficients on the infiltration and ventilation rates is investigated. Further research dealing with uncertainty is reported in (Holm and Kuenzel, 2002), where the 
authors investigated the impacts of materials properties and surface coefficients on hygrothermal building simulation using a Monte Carlo technique.

More recently, in (Corrado and Mechri, 2009) a sensitivity analysis of the quasi steady state approach proposed by EN ISO 13790:2008 (CEN, 2008) is proposed for the calculation of the energy performance for heating. Similarly, Tian and deWilde (2011) explored the uncertainties of climate, construction material properties, infiltration rates, internal loads and equipment efficiency for building simulations of an office building in the UK.

In (Hopfe and Hensen, 2007) and in (Hopfe and Hensen, 2011), the influence of uncertainty in the early stage of design process is analyzed, while, in (Dominguez-Munoz, Cejudo-Lopez, and CarriloAndres, 2011) a study about the impacts of suboptimal design parameters on the simulated peak-cooling loads is presented.

Often in the literature, the authors analyze the simultaneous effect of several parameters on the simulations outcomes. However, due to their different nature, it is dangerous to combine different sorts of uncertainties (Hopfe and Hensen, 2011). Furthermore, since the energy behaviour is not always linear, in order to draw reliable considerations from results, a realistic distribution of the input data is required. Unfortunately, the literature review shows there are limited data available describing uncertainties for design parameters in building energy simulation.

For these reasons, this work focuses on the effects of perturbations in the thermo-physical properties on the numerical solutions of the heat transfer equation. In particular, the primary goal is the investigation of the robustness of different calculation procedures such as finite difference method (FD) and conduction transfer functions (CTF) when suboptimal thermo-physical properties are used. Besides, this work aims to clarify if different calculation procedures can respond in different ways to perturbed input data. To this purpose, the analysis focuses on the heat transfer through the envelope (i.e. inward and outward heat losses) computed both with FD and CTF approaches, when Gaussian distributions of the material thermo-physical properties are imposed.

\section{Calculation procedure}

\subsection{The Monte Carlo method}

In this paper, the sensitivity analysis of the calculation procedures for the numerical solution of the heat transfer partial differential equation is carried out using the Monte Carlo method. The idea behind the Monte Carlo approach is that if a set of data with the same statistic of the population can randomly be found, these data can be used as deterministic input in the heat transfer model and, subsequently, the distribution of the model expectation could be generated (Fig. )

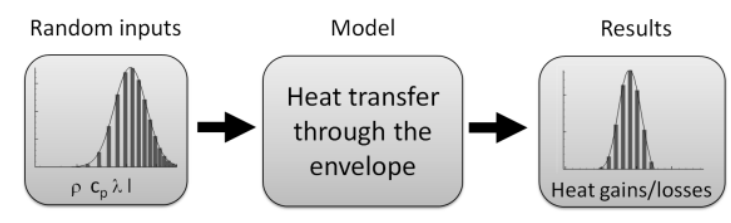

Fig. 1 - Scheme of research analysis carried on

A Monte Carlo method entails full random selection, out of all possible values of the inputs in a correct statistical combination. When the selection of data is improved, the number of simulations can be reduced (Macdonald, 2002). However, with enhanced sampling methods, unbiased results cannot be taken for granted and, consequently, it should be verified with additional computational costs. Therefore, in this work a simple random sampling method is adopted (Prada, 2012). It is the most basic method, which works by generating random numbers in agreement with the probability distribution and scaling this to the target value. The results are an unbiased estimation of the population variance.

\subsection{The numerical solution of heat transfer equation}

Since the analytical solution of heat equation is known only for simple domain and boundary conditions, in building simulations numerical methods are adopted. Among the possible ways to 
numerically solve the partial differential equation, the main strategies are the domain discretization and the use of a frequency analysis for the calculation of the time series terms, which are called conduction transfer function.

The key obstacle in using a finite difference approach (FD) is finding a stable and not time consuming procedure. In this work, with the purpose of ensuring the stability of the solution, a semi-implicit scheme (i.e. Crank-Nicholson) is adopted. However, building thermal simulation software uses to a large extent the CTF method for the evaluation of transient heat conduction. Despite the simplicity of the equations, the complexity of the procedure lies in the evaluation of the CTF coefficients. Several calculation techniques have been proposed. Nevertheless, those that are largely diffused are the Direct Root Finding approach (Hittle, 1979) and the State Space method (Seem, 1987). In this paper the DRF method is adopted for the analysis with perturbed thermo-physical properties. Both the calculation procedure and the Monte Carlo routine are implemented by means of a Fortran code.

\subsection{Boundary conditions}

The heat transfer transmitted through the envelope is evaluated performing a hourly simulation over an annual period. With the FD approach a variable spatial discretization $(\max 3 \mathrm{~cm})$ is chosen. Moreover, even if the Von Neumann stability analysis shows that semi-implicit scheme is unconditionally stable, a time discretization, which ensures to meet the condition Fourier number lower than 0.5, is herein adopted. In order to numerically solve the partial differential equation, initial and boundary conditions are imposed. While the steady state solution at the starting time is adopted as initial values, hourly temperatures are imposed on the external environment. In particular, in order to take into account the effect of both convective and radiative exchanges, the temperature adopted is the so-called sol-air temperature (ASHRAE, 2009). The sol-air temperature is a simplification that allows to greatly reduce the computational cost of the dynamic simulations and it can be computed as:
$T_{\text {solair }}=T_{d b}+\frac{\alpha \cdot G_{\text {solar }}}{h_{0}}-\frac{\varepsilon \cdot \Delta R}{h_{0}}$

where $\Delta R$ is the difference between long-wave radiation incident on surface from sky and surroundings and radiation emitted by blackbody at outdoor air temperature. Because of the multiple sources of long-wave radiation, for vertical surfaces accurate $\Delta R$ values are difficult to determine. For instance, in (Gasparella et al. 2011) the predicted heat fluxes obtained by means of hourly dynamic simulations are used for the estimation of the sol-air temperature. However, according to the ASHRAE Handbooks (ASHRAE, 2009) when solar radiation intensity is high, surfaces of terrestrial objects usually have a higher temperature than the outdoor air. Thus, their longwave radiation compensates to some extent for the long-wave radiation to the sky. Since in this work a vertical south orientated wall is analysed, a $\Delta R$ equal to zero is adopted. Besides, the other parameters are the surface absorptance equal to 0.6 and $h_{0}$ of $17 \mathrm{~W} \mathrm{~m}^{-2} \mathrm{~K}^{-1}$. Starting from the test reference year (TRY) for the analyzed cities, the hourly values of the sol-air temperature are therefore computed and used in the external boundary conditions. On the inner surface a RobinNewton condition is used by assigning a surface convective and long wave radiation coefficient (i.e. $7.69 \mathrm{Wm}^{-2} \mathrm{~K}^{-1}$ ) and a fixed internal temperature (i.e. $20{ }^{\circ} \mathrm{C}$ for winter and $26{ }^{\circ} \mathrm{C}$ for summer). In order to have a consistent comparison between the calculation procedures, similar boundary conditions are imposed also in the CTF approach. Therefore the air to air CTFs are evaluated by introducing the additional resistive layers at the internal and external surfaces. Then, the heat transfer through the envelope, using the sol-air and the internal fixed temperature, is computed.

\subsection{The test cases}

The choice of the envelope typology is a key point in the uncertainty analysis. Besides, since the unsteady heat conduction through the envelope is strictly connected to the thermal inertia of the component, also the arrangement of layers has an important role in the envelope dynamic behaviour. 
The most common types of building envelope in Italy are investigated and adopted. In particular, in Italy about $20 \%$ of the housing stock was built before the $20^{\text {th }}$ century. In this period the construction type is various and includes brick and stone walls (Fig. 2). The latter (SW case), due to the high thickness and mass, is of particular interest to the uncertainty analysis. While the SW case is representative of existing buildings, for new constructions, which must meet energy requirements, the typology most widely diffused in low rise residential buildings is the ME case This opaque structure is a multilayer wall with external insulation (Fig. 3). For both test cases, reasonable physical properties are chosen, by analysing either the manufacturer technical sheets or reference standard values. The properties adopted and used in this work are reported in Table .

In order to ensure a greater representativeness of the Monte Carlo simulation results, two different climates are analysed. More specifically, Trento, and Palermo test reference years are used with the purpose of modelling both warm and mixed climates. The coordinates and the main characteristics of the sites used in the Monte Carlo simulations are shown in Table 2. Since the work focuses on the effect of suboptimal input data, one of the key aspects, in order to obtain meaningful results from the stochastic simulation, is the quantification of the input variability. Besides, it should not be forgotten that there is a strong correlation between the material properties. For example, moisture content would impact upon the conductivity, density and specific heat capacity simultaneously. Further the specific mass for insulation material is strictly connected to conductivity as shown in (Dominguez-Munoz et al., 2010). However, in this study the cross correlations are not taken into account and each thermo-physical property is treated as an independent variable. A realistic quantification of the parameter variability can become difficult and time consuming due to the lack of information.

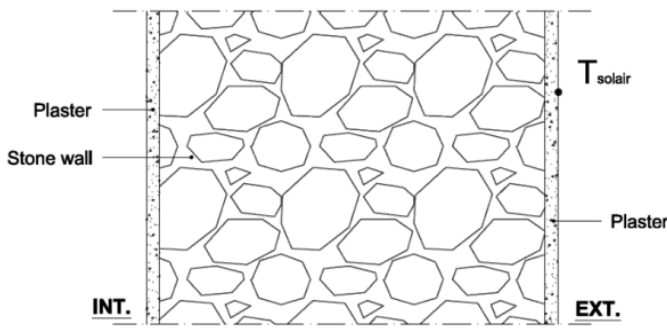

Fig. 2 - Poorly insulated wall (SW case)

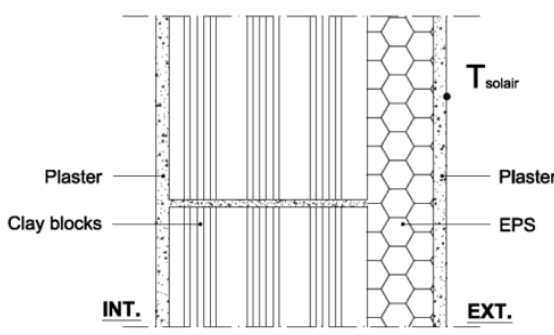

Fig. 3 - Well insulated wall (ME case)

\begin{tabular}{|l|l|l|l|l|}
\hline Layer & $\lambda$ & $\rho$ & $\mathrm{c}_{\mathrm{p}}$ & 1 \\
\hline $\begin{array}{l}\text { Internal } \\
\text { Plaster }\end{array}$ & 0.70 & 1400 & 1010 & 0.01 \\
\hline Clay Blocks & 0.26 & 600 & 840 & 0.30 \\
\hline Insulation & 0.036 & 30 & 1500 & 0.10 \\
\hline Stone wall & 2.20 & 2000 & 1000 & 0.70 \\
\hline $\begin{array}{l}\text { External } \\
\text { Plaster }\end{array}$ & 0.90 & 1800 & 910 & 0.02 \\
\hline
\end{tabular}

Table 1 - Average values of adopted properties

\begin{tabular}{|l|l|l|l|l|}
\hline City & Lat. & Elev. & HDD $_{20}$ & CDD $_{20}$ \\
\hline Trento & $\begin{array}{l}46^{\circ} .02 \\
\mathrm{~N}\end{array}$ & $185 \mathrm{~m}$ & 2798 & 197 \\
\hline Palermo & $\begin{array}{l}38^{\circ} .13 \\
\mathrm{~N}\end{array}$ & $50 \mathrm{~m}$ & 994 & 704 \\
\hline
\end{tabular}

Table 2 - Climate feature of reference cities

In fact, handbooks, data sheets and technical standards provide safe values but they do not usually declare the variability of these characteristics. Besides, in the literature only a few works have formally dealt with the issue of uncertainty (e.g. Dominguez-Munoz et al., 2010). For this study, a Gaussian distribution of thermophysical properties is used. The normal distribution is adopted in order to correctly represent the variability of material characteristic caused by random errors. Mean and variance are 
defined for each material based on the comparison between manufacturer data sheets and on data reported in the literature (Prada, 2012). The variability of each material property is herein summarized in Table 3, where the $1 \%$ and $99 \%$ fractiles are highlighted.

\begin{tabular}{lrrr}
\hline Layer & Clay Blocks & Insulation & Stone wall \\
\hline$\lambda$ & $0.17 \div 0.36$ & $0.031 \div 0.041$ & $1.75 \div 2.70$ \\
\hline$\rho$ & $510 \div 700$ & $26.5 \div 33.5$ & $1551 \div 2503$ \\
\hline$c_{p}$ & $620 \div 1090$ & $1060 \div 2000$ & $670 \div 1370$ \\
\hline 1 & $0.28 \div 0.31$ & $0.08 \div 0.12$ & $0.59 \div 0.83$ \\
\hline
\end{tabular}

Table 3 - Fractiles $1 \%$ and $99 \%$ of distributions

\subsection{Postprocessing of results}

The last step in the analysis involves the investigation of the monthly heat losses distribution. For both cities, January and August are identified as the most representative months respectively for outward and inward heat losses. To do this analysis, a postprocessing code is implemented by means of Matlab.

Starting from the hourly values of transmitted heat flux through the envelope, computed either with FD or CTF approaches, the terms are firstly separated in inward heat losses and outward heat loss contributions and assigned to one of the 90 bins in which the output variability range is divided.

Finally, the code fits one of three probability distributions (i.e. Normal, Log-normal and Weibull) to the output data distributions. The distribution that best fits the data is selected automatically. assuming The sum of point by point square differences between outputs and the values of each distribution function is assumed as measure of goodness of fit. The lower the squared 2-norm of residual, the better the fit. The code estimates the parameters of the curve that best approximate the actual output distribution, and in particular $\mu$ and $\sigma$ for normal and lognormal distribution or $\alpha$ and $\beta$ for the Weibull distribution.

\section{Results and Discussions}

\subsection{Shapes of probability density functions (PDF)}

As a first step in the result comparison, the PDF shapes are herein presented. The PDF shape analysis firstly highlights that the use of Gaussian random fields as inputs does not imply a Gaussian structure of the heat loss distributions. The distortion of the PDF shape may be represented as a propagation of probability distributions through the model, and the uncertainty is propagated through the DRF and FD methods. In fact, while thickness uncertainty always induces a normal distribution of the simulation outcomes, a perturbation of material conductivity causes a Weibull distribution. Nonetheless, due to the low skewness of these Weibull distributions, the result PDFs are close to normal distributions.

Otherwise, when the perturbed parameters are either specific heat or specific mass, a preponderance of asymmetrical curves, in particular log-normal distributions, is noted. It should be stressed that the shapes of the distributions vary according to the wall typology and to climate conditions. In any case the perturbation of either specific mass or specific heat induces the same output distributions.

\subsection{Sensitivity of calculation procedures}

As is to be expected, the CTF and FD methods give consistent results with deviations within $\pm 5 \%$ when deterministic inputs are used. Based on this result, this work investigates the extent to which CTF and FD methods are sensitive to the perturbation of the input data. Both calculation procedures show similar responses to the perturbation of the material thermo-physical parameters. Indeed, similar results are obtained both in the shape of the PDF and in the moment of distributions, e.g. the expected values and the variances (Fig. 4a and Fig. 4b).

However, Fig. 4c and Fig. 4d show a greater sensitivity of the CTF approach with respect to the FD method when an uncertain specific mass is used as input data. This result, however, is found only in the SW case, which is a massive wall 
characterized by a high thickness. Therefore, this higher sensitivity is probably related to the wellknown numerical issues associated with the root searching in the Laplace space of the Direct Root Finding method used in the CTF approach. Since a substantial agreement of the methods has been

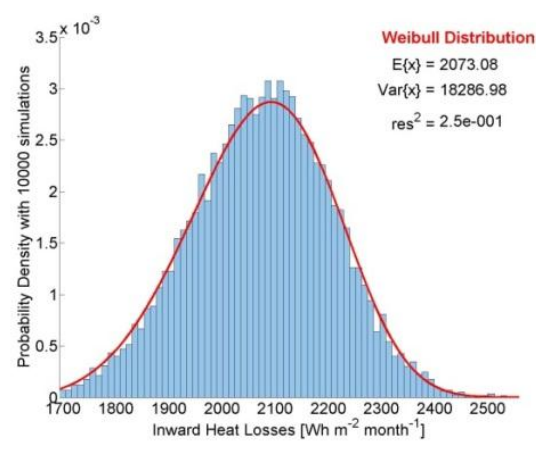

$\lambda$ perturbations with CTF method in Trento

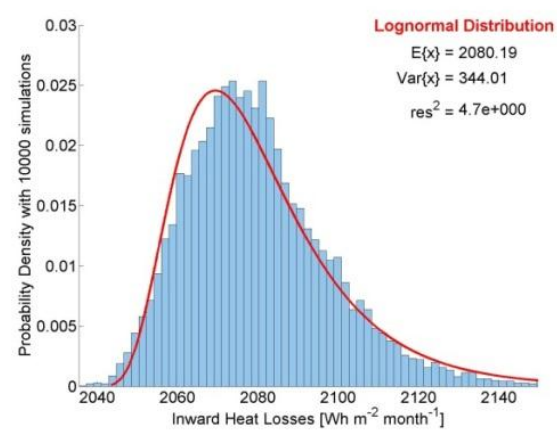

$\rho$ perturbations with CTF method in Trento proved by comparing the monthly inward and outward heat losses computed for several wall typologies and climate conditions, the following considerations are herein presented only for the finite difference method. However, similar results are found for the CTF calculation procedure.

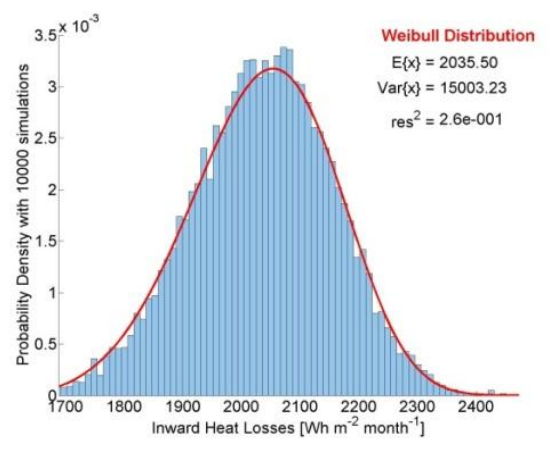

$\lambda$ perturbations with FD method in Trento

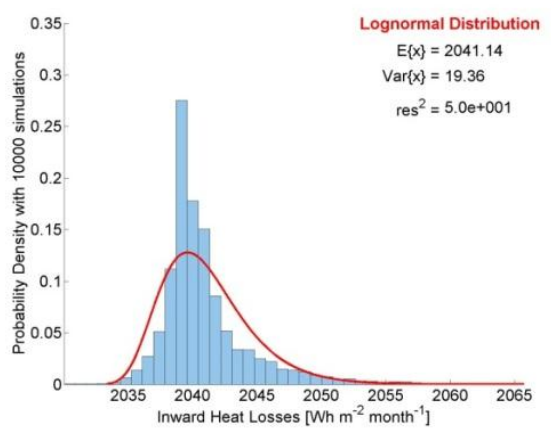

$\rho$ perturbations with FD method in Trento

Fig. 4 - August output PDF of case SW in Trento as a function of calculation procedure.

\subsection{Influence of climate conditions on PDF shape}

The second part of the comparison focuses on the investigation of the interaction between climate and uncertainty in the thermo-physical parameters. For this reason, two different climates are investigated with the purpose of quantifying the extent to which a higher fluctuation of the sol-air temperature around the internal setpoint can affect the reliability of the simulation outcomes.

In Fig. 5 the PDF curves obtained for the ME case when uncertain specific mass is used either in Trento or Palermo are presented. Notice that there is an opposite change in the PDF shapes in Trento with respect to Palermo. In fact, while in Trento the
Gaussian distribution of the inward heat losses in the winter months becomes skewed for the outward heat losses in August, in Palermo an opposite trend is registered. This can be explained taking into account the role of the specific mass. In fact, specific mass together with specific heat assume a primary role in the heat transfer because of describing the capability of the wall to work as a thermal storage. Nevertheless, if the oscillation of the sol-air temperature around the internal set-point is limited, the heat flux has a prevailing direction and the effect of accumulation is less. This is what happens for example in the winter months in colder climates (e.g. Trento) and in the summer months in warm climates (e.g. Palermo). In these cases, therefore, the effect of perturbed specific mass on the heat capacitance are 
not highlighted and, consequently, the output does not assume the characteristic log-normal distribution that instead occurs when the role of the thermal capacitance grows (Fig. 5). These changes in PDF shapes due to variations in climate conditions are not found instead for the perturbations of either thermal conductivity or thickness. In fact, these characteristics primarily affect the thermal resistance of the wall and, consequently, the stationary part of the solution of the heat transfer problem.

\subsection{Spread comparison of model predictions}

With the purpose of comparing the sensitivity of the models to the perturbation of different input, the same variability of thermo-physical properties is required. Although this is not strictly observed in this analysis, the ranges of percentage variability of the different parameters used are similar (within \pm $30 \%$ ) and therefore a preliminary and qualitative analysis can be carried out. In order to better graphically represent the variability of the heat losses through the envelope, a box plot is adopted. The box plot leads to describe the degree of dispersion and skewness in the distribution of simulation outcomes by means of the simultaneous representation of the $1 \%$ fractiles of observation, lower quartile, median, upper quartile and the $99 \%$ fractiles of observation. Besides, with the aim of directly comparing the results obtained in different climate conditions and in different test cases, a normalization of the results is adopted.

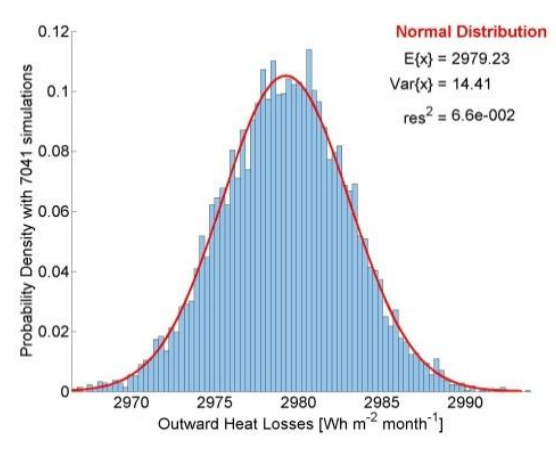

$\rho$ perturbations in Trento (January)

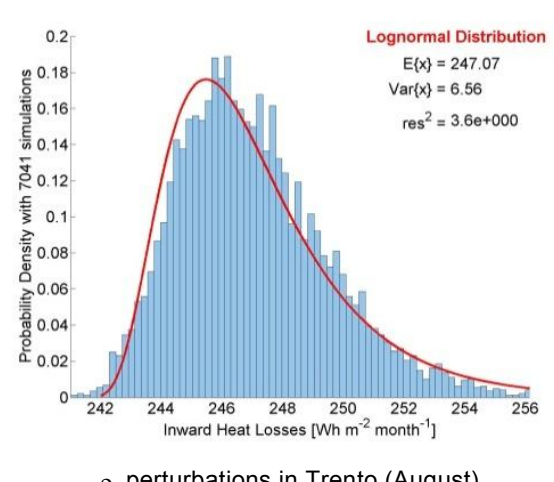

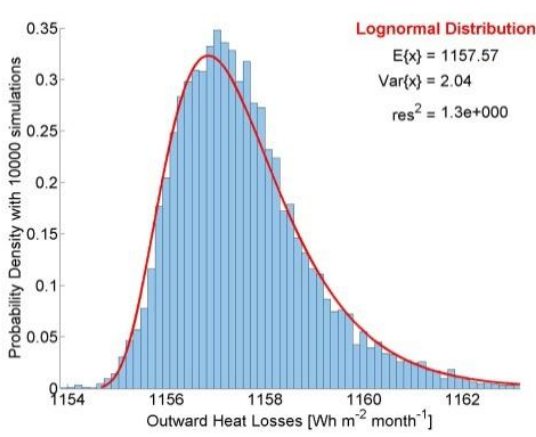

$\rho$ perturbations in Palermo (January)

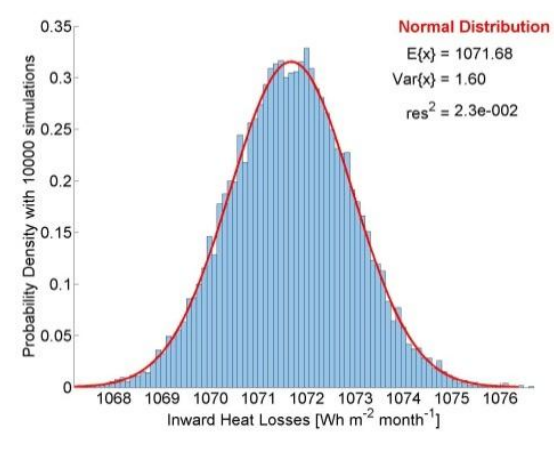

$\rho$ perturbations in Palermo (August)

Fig. 5 - Output PDF of case ME with FD approach as a function of climate conditions.

In particular, the perturbed heat losses have been divided by the unperturbed one, thus highlighting the differences from predictions of the model with deterministic input.

The box plots in Fig. 6a and Fig. 6b show the spread of the predicted monthly inward heat losses in
August of the ME test, when suboptimal input data are used respectively for clay blocks and insulation layer. For both figures notice that a negligible dependence of normalized heat losses on specific mass and on specific heat is found. Only in Trento, due to the high daily temperature range, is there a 
light influence of specific mass and specific heat with respect to clay block while, of course, variations of these parameters on the insulation layer are negligible.

Moreover, while in clay block contained variations emerge due to the perturbation of layer thickness, the FD model seems to be more sensitive to insulation thickness. In reality this is mainly caused by the effect of thickness perturbation on the total thermal resistance of the element. In fact, while the variation of the insulation thickness alters by about $\pm 14 \%$ the total resistance, the clay bocks change it by only $\pm 2 \%$. Instead, the uncertainties of thermal conductivity induce a variation of the total thermal resistance between $\pm 10 \%$ for both materials and this produces an equal sensitivity of the method and, consequently, an equal data dispersion.

The graph in Fig. 6a also shows how, with increasing amplitude of daily fluctuations of sol-air temperature, around the internal set-point temperature (e.g. in Trento), also the results variability caused by the perturbations of either the thickness or the thermal conductivity increases. This is because the thickness affects both the thermal resistance and the thermal capacitance of the wall. As well as the thermal conductivity, perturbation produces an alteration of the thermal diffusivity, which is the main parameter in the description of the transient part of the solution of heat transfer problem. A similar comparison is also carried out for the SW case and the results are shown in Fig. 6c.

In contrast with the ME case with uncertain clay block properties, no significant variations of the normalized inward heat losses are noted between Trento and Palermo. In addition, notice that despite the similar percentage perturbations of specific mass and specific heat in the ME case, it produces less spread in predicted inward heat losses (Fig. 6c). This is probably caused by the high thermal capacitance of the SW wall, which may result much higher than the capacitance required to accumulate and release the daily variations of heat flux.

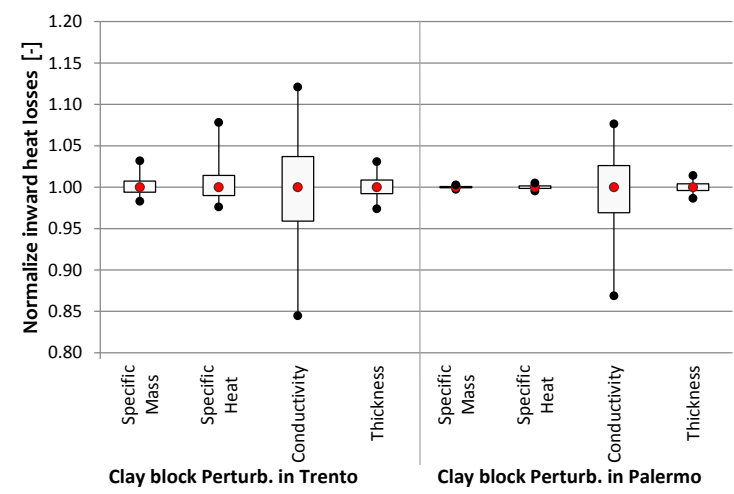

Uncertain clay block properties (case ME)

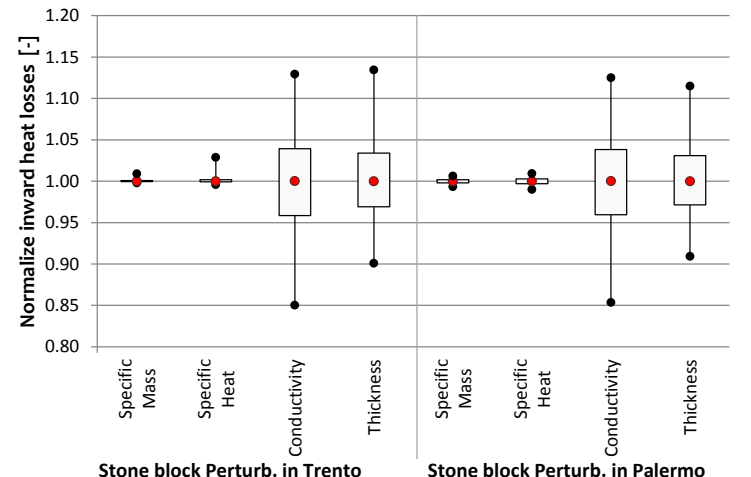

Uncertain stone block properties(case SW)

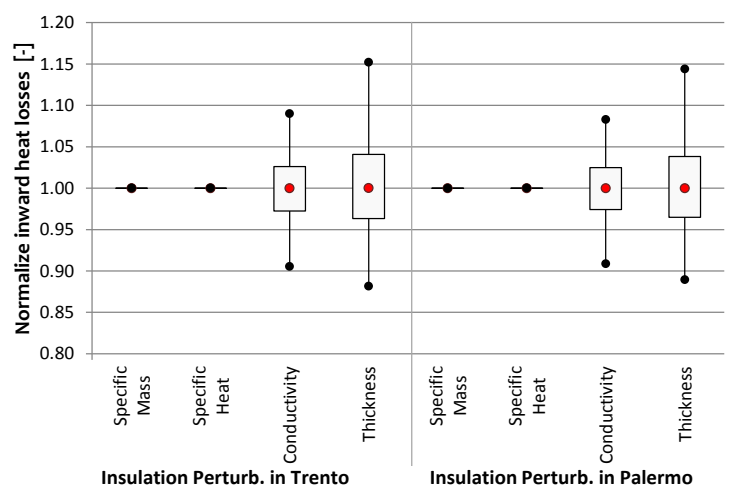

Uncertain insulation properties (case ME)

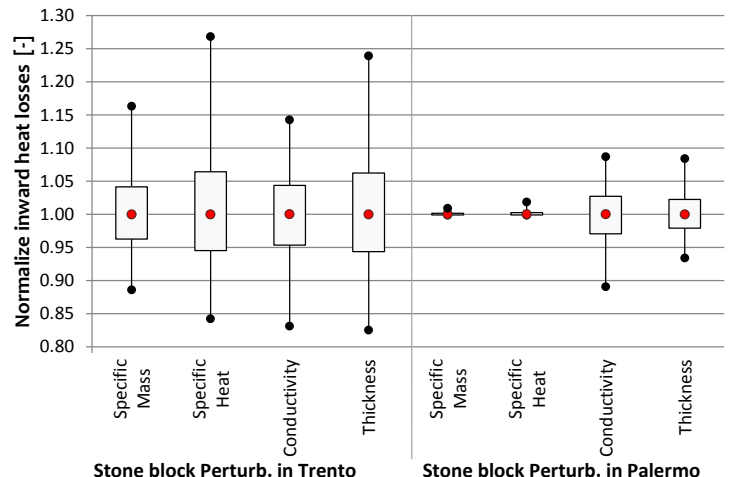

Uncertain stone properties (low thickness layer)

Fig. 6 - Normalized heat losses(FD approach) due to uncertain clay block properties (case ME). 
Therefore, even if the wall behaviour is greatly influenced by the thermal capacitance, the perturbations of specific mass and specific heat do not induce changes in the used thermal capacitance. This is confirmed by the analysis carried out on a wall similar to the SW case, in which, however, the thickness of the stone layer is $30 \mathrm{~cm}$. The other thermo-physical parameters and the percentage perturbation of the input data are kept unchanged with respect to the SW case. Fig. 6 d stresses the role of specific mass and specific heat perturbations in temperate climates such as Trento. The box plot shows how the variability on the inward heat transfer in Trento, due to either uncertain specific mass or specific heat, becomes comparable to those caused by the conductivity and thickness perturbations. Because of the warmer climate, this does not happen in Palermo. Therefore the weight of the heat capacitance in the wall thermal response decreases and, consequently, the role of the perturbations imposed on specific mass and specific heat drops off.

The results obtained in this work are summarized in Table 4, where the distance between the upper and lower quartile (i.e. interquartile range) of the inward heat losses distributions is reported for each test case and for each uncertain parameter.

\begin{tabular}{|c|c|c|c|c|c|c|}
\hline & & & $\begin{array}{c}\text { Specific } \\
\text { Mass }\end{array}$ & $\begin{array}{c}\text { Specific } \\
\text { Heat }\end{array}$ & Conductivity & Thickness \\
\hline \multirow{4}{*}{ sw } & \multirow{2}{*}{ Trento } & CTF & $4.26 \%$ & $7.39 \%$ & $30.19 \%$ & $27.29 \%$ \\
\hline & & FD & $1.12 \%$ & $3.29 \%$ & $27.91 \%$ & $23.36 \%$ \\
\hline & \multirow{2}{*}{ Palermo } & CTF & $0.07 \%$ & $0.11 \%$ & $27.92 \%$ & $22.10 \%$ \\
\hline & & FD & $1.29 \%$ & $1.91 \%$ & $27.15 \%$ & $20.57 \%$ \\
\hline \multirow{4}{*}{$\begin{array}{c}\text { ME } \\
\text { clay } \\
\text { block } \\
\text { perturb. }\end{array}$} & \multirow{2}{*}{ Trento } & CTF & $5.15 \%$ & $10.53 \%$ & $28.37 \%$ & $5.80 \%$ \\
\hline & & FD & $4.88 \%$ & $10.21 \%$ & $27.65 \%$ & $5.70 \%$ \\
\hline & \multirow{2}{*}{ Palermo } & CTF & $0.01 \%$ & $0.03 \%$ & $21.59 \%$ & $3.04 \%$ \\
\hline & & FD & $0.54 \%$ & $0.96 \%$ & $20.77 \%$ & $2.74 \%$ \\
\hline \multirow{4}{*}{$\begin{array}{c}\text { ME } \\
\text { insulatio } \\
n \\
\text { perturb. }\end{array}$} & \multirow{2}{*}{ Trento } & CTF & $0.01 \%$ & $0.04 \%$ & $18.29 \%$ & $26.86 \%$ \\
\hline & & FD & $0.02 \%$ & $0.04 \%$ & $18.47 \%$ & $27.04 \%$ \\
\hline & \multirow{2}{*}{ Palermo } & CTF & $0.01 \%$ & $0.01 \%$ & $17.54 \%$ & $25.64 \%$ \\
\hline & & FD & $0.01 \%$ & $0.04 \%$ & $17.44 \%$ & $25.48 \%$ \\
\hline \multirow{4}{*}{$\begin{array}{l}\mathrm{SW} \text { with } \\
\mathrm{L}=\mathbf{3 0} \mathrm{cm}\end{array}$} & \multirow{2}{*}{ Trento } & CTF & $25.81 \%$ & $39.98 \%$ & $30.28 \%$ & $39.51 \%$ \\
\hline & & FD & $27.73 \%$ & $42.60 \%$ & $31.15 \%$ & $41.42 \%$ \\
\hline & \multirow{2}{*}{ Palermo } & CTF & $4.43 \%$ & $6.80 \%$ & $21.28 \%$ & $18.45 \%$ \\
\hline & & FD & $1.03 \%$ & $2.01 \%$ & $19.62 \%$ & $15.02 \%$ \\
\hline
\end{tabular}

Table 4 - Percentage interquartile range for inward heat losses

\section{Conclusions}

The present work investigates the extent to which uncertain thermo-physical properties of envelope materials can affect the reliability of predicted heat transfer through the envelope.

Firstly, the comparison between the Finite Different method and the Conduction Transfer Function approach demonstrates that the two calculation procedures provide consistent results both with deterministic and uncertain parameters. Only a little deviation in sensitivity to either specific mass or specific heat is registered for the massive envelope. This is probably caused by the numerical issues in the Direct Root Finding technique.

Secondly, the results of Monte Carlo simulations reveal that the outcomes of calculation procedures are not always normally distributed when Gaussian distributions of input data are adopted. This skewness is highlighted especially for perturbed specific mass or specific heat. Moreover, these parameters show a greater correlation with the external climate conditions. In particular, since specific mass and specific heat primarily affect the thermal capacitance of the wall, the output uncertainty is emphasized by the amplitude of daily oscillation of the sol-air temperature around the internal set-point temperature. However, for the SW case the sensitivity to the perturbations of specific mass and specific heat is smoothed over. In fact, the uncertainty of these parameters significantly affects the results only when the thermal capacitance needed to absorb the daily changes in sol-air temperature is modified.

For non-insulated but high massive walls, although the behaviour is mainly governed by the thermal capacitance, the uncertainties of specific mass and specific heat have limited influence on the results.

\section{Nomenclature}

\section{Symbols}

$\begin{array}{ll}\mathrm{C}_{\mathrm{p}} & \text { Specific Heat }\left(\mathrm{J} \mathrm{kg}^{-1} \mathrm{~K}^{-1}\right) \\ \mathrm{CDD}_{20} & \text { Cooling degree days base on } 20^{\circ} \mathrm{C} \\ \mathrm{E}\{\mathrm{x}\} & \text { Expected value of } \mathrm{x} \\ \mathrm{G}_{\text {solar }} & \text { Global solar irradiation }\left(\mathrm{W} \mathrm{m}^{-2}\right) \\ \mathrm{HDD}_{20} & \text { Heating degree days base on } 20^{\circ} \mathrm{C} \\ \mathrm{L} & \text { Layer thickness }(\mathrm{m}) \\ \mathrm{T}_{\mathrm{db}} & \text { Dry Bulb Temperature }(\mathrm{K})\end{array}$




$\begin{array}{ll}\operatorname{Var}\{\mathrm{x}\} & \text { Variance of the } \mathrm{x} \text { variable distribution } \\ \mathrm{h}_{0} & \text { Heat transfer coefficient }\left(\mathrm{W} \mathrm{m}^{-2} \mathrm{~K}^{-1}\right) \\ \mathrm{res}^{2} & \text { Residuals 2-norm of the fitting } \\ \mathrm{x} & \text { Random variable }\end{array}$

\section{Greek symbols}

$\begin{array}{ll}\alpha & \text { Wall solar absorptance (-) } \\ \varepsilon & \text { Wall hemispherical emissivity (-) } \\ \lambda & \text { Thermal conductivity }\left(\mathrm{W} \mathrm{m}^{-1} \mathrm{~K}^{-1}\right) \\ \rho & \text { Specific mass }\left(\mathrm{kg} \mathrm{m}^{-3}\right)\end{array}$

\section{References}

ASHRAE. (2009). ASHRAE HandbookFundamentals (SI Edition). Atlanta (USA): American Society of Heating, Refrigerating and Air-Conditioning Engineers, Inc.

CEN. (2008). EN ISO 13790. European Committee for Standardization.

Corrado, V., and Mechri, H. (2009). Uncertainty and sensitivity analysis for building energy rating. Journal of building physics, 33(2), 125-156.

Costola, D., Blocken, B., and Hensen, J. (2009). Overview of pressure coefficient data in building energy simulation and airflow network programs. Building and Environment, 44(10), 2027-2036.

Dominguez-Munoz, F., Anderson, B., Cejudo-Lopez, J., and Carrillo-Andres, A. (2010). Uncertainty in the thermal conductivity of insulation materials. Energy and Buildings, 42(11), 21592168.

Dominguez-Munoz, F., Cejudo-Lopez, J., and Carrilo-Andres, A. (2011). Uncertainty in peak cooling load calculations. Energy and Buildings, 42(7), 2798-2805.

Gasparella, A., Pernigotto, G., Baratieri, M. and Baggio, P. (2011). Thermal dynamic transfer properties of the opaque envelope: analytical and numerical tools for the assessment of the response to summer outdoor conditions. Energy and Buildings, 43, 2509-2517.

Hittle, D. (1979). Building loads analysis and system thermodynamics program BLAST user's manual. Champaign, Illinois (USA): U.S. Army Construction Engineering Research Laboratory.

Holm, A., and Kuenzel, H. (2002). Practical application of an uncertainty approach for hygrothermal building simulations-drying of an AAC flat roof. Building and Environment, 37(8-9), 883-889.

Hopfe, C., and Hensen, J. (2007). Uncertainty analysis for building performance simulation a comparison of four tools. Building Simulation, (pp. 1383-1388). Beijing (China).

Hopfe, C., and Hensen, J. (2011). Uncertainty analysis in building performance simulation. Energy and Buildings, 43(10), 2798-2805.

Lomas, K., and Eppel, H. (1992). Sensitivity analysis techniques for building therma simulation programs. Energy and Buildings, 19(1), 21-44.

Macdonald, I. (2002). Uncertainty in building simulations. Glasgow UK: University of Strathclyde.

Prada, A. (2012). Energy performance of buildings: modeling of dynamic summer behavior. Ph.D. Thesis, University of Trento, Civil and Environmental Engineering, Trento.

HYPERLINK "http://eprintsphd.biblio.unitn.it/770/" http://eprintsphd.biblio.unitn.it/770/

Seem, J. (1987). Modeling of heat transfer in buildings. Ph.D. Thesis, Madison, Wisconsin (USA).

Tian, W., and deWilde, P. (2011). Uncertainty, sensitivity analysis of building performance probabilistic climate projections: a UK case study. Automation in construction, 20(8), 10961109. 